\title{
Contemporary Definitions of Hypoglycemia as Frequent and Dangerous Syndrome and Coma
}

\author{
Vyacheslav Semionovish Lukyanchikov
}

Medical Centre "Medstyle-Effect", Moscow, Russia

Email address:

vsluk@yandex.ru

\section{To cite this article:}

Vyacheslav Semionovich Lukyanchikov. Contemporary Definitions of Hypoglycemia as Frequent and Dangerous Syndrome and Coma. American Journal of Life Sciences. Vol. 7, No. 5, 2019, pp. 83-90. doi: 10.11648/j.ajls.20190705.11

Received: September 27, 2019; Accepted: October 17, 2019; Published: October 29, 2019

\begin{abstract}
A decrease in blood glucose is a frequent and dangerous violation of the most important part of homeostasis energy. The high frequency of hypoglycemia is explained by a pathogenetic relationship with many diseases and syndromes, as well as the important role of hypoglycemia as a physiological regulator of energy metabolism. The danger of hypoglycemia is that glucose is actually the only source of energy for nerve structures and a lack of glucose could lead to their damage. In addition, deep hypoglycemia and low glucose concentration in brain cells could lead to cerebral edema. Analyzes of the respectful literature for a 20-year period, as well as my own clinical experience, including the work as an endocrinologist surgeon. The article presents the definitions of hypoglycemia, the typology of the syndrome in terms of causes, development mechanisms and clinical forms, also issues of the clinic and laboratory diagnostics are defined. Algorithms for the treatment of hypoglycemic coma and pathogenetic diagnosis of the main variants of hypoglycemia are proposed. Hypoglycemic syndrome [HS] and hypoglycemic coma [HC] are frequent clinical and laboratory paroxysms, especially among patients with diabetes mellitus. These conditions are of great danger to the health and even to the lives of patients, and require quick, qualified help.
\end{abstract}

Keywords: Hypoglycemia, Glycopenia, Hypoglycemic Coma, Urgent Endocrinopathies

\section{Introduction}

Endocrine and metabolic disorders are crucial component of many pathological processes. Most often, these are disorders of carbohydrate metabolism in the form of an excessive increase (hyperglycemia), or a deep decrease (hypoglycemia) in the blood glucose level. Such disorders often become the leading pathobiochemical factor for some disease or syndrome, giving them a peculiar and vivid clinical symptom. It should be noted that glycemic disorders are not always a pathology. Often, they are transient, selfstopping in nature, in the form of an inadequate adaptation reaction, which temporarily went beyond the physiological norm. These characteristics are most common for hypoglycemia, or to be more precise, for hypoglycemic syndrome (HS), which is not only a frequent pathological phenomenon, but also the most important physiological regulator of energy metabolism. Therefore, it is very difficult, but important, to clearly define and classify the HS.

\section{Methods}

In the frame of the article preparation the following methods were applied: the meta-analysis of the thematic literature for a 40-year period, the analysis of personal clinical observations obtained over a long period of work in the departments of surgical and therapeutic endocrinology in a number of Moscow multidisciplinary hospitals. Based on the data obtained: 1. The definitions of $\mathrm{HS}$ and $\mathrm{HC}$ are formulated. 2. Laboratory definitions and clinical characteristics of HS are updated. 3. A typological assessment of the HS is undertaken in the form of two working classifications. 4. Etiological and pathogenetic characteristics of the most frequent forms of HS are presented. 5. Algorithms of urgent therapy of $\mathrm{HC}$ and schemes of etiological and pathogenetic diagnostics of HS are offered. These results are presented below. 


\section{Results}

\subsection{Definitions and Classification of Hypoglycemia}

Hypoglycemic syndrome (HS) is a laboratory-clinical complex of symptoms caused by a decrease in blood glucose concentration below the physiological minimum, or by a rapid drop in glycemia from a very high to significantly lower level, which is accompanied by polymorphic and vivid clinical symptoms.

Hypoglycemic coma (HC) is an urgent clinical syndrome that occurs against a background of a significant or very rapid decrease in blood glucose, resulting in severe impairment of consciousness or its loss.

Definitions of HS and HC consist of two conceptions: "chemical hypoglycemia" - the result of laboratory analysis, which records a drop-in blood glucose below the physiological norm, and "clinical hypoglycemia", which implies a complex of subjective and physical symptoms. Unfortunately, the lower limit of normoglycemia in physiological conditions is a subjective value, with wide individual and age-related variations. Still, this value depends on the laboratory method used for determining glucose and the choice of the research environment - arterial, venous, capillary blood, blood plasma. When measuring glycemia in capillary blood with orthotoluidine or enzymatic methods, the lower limit of the norm is fixed at $2.7-2.8 \mathrm{mmol} / \mathrm{L}$, and individual and age-related variations of this indicator are \pm $10 \%$ or $0.28 \mathrm{mmol} / \mathrm{L}$. Thus, "chemical hypoglycemia" is a decrease in the concentration of glucose in the blood by at least $10 \%$ of the lower limit of the norm adopted in this laboratory for this laboratory method and for a specific environment $[1,2]$.

To formulate the concept of "clinical hypoglycemia" is even more difficult. The fact is that when the glucose level in the blood only approaching the lower limit of the norm, it provokes the excitation of the central nervous system, somatic and autonomic nervous system, and also stimulates the secretion of a number of systemic hormones, especially "stress-hormones" - catecholamines, cortisol, glucagon. This physiological reaction is manifested by a "feeling of hunger", which is typical for HS. During this period energy metabolism switches from digestion to endogenous energy sources, while the decrease in blood glucose levels is a trigger, up to the development of "functional chemical hypoglycemia" (see below).

The main definitive and typological complexity of HS is that there is no strict correlation between chemical and clinical hypoglycemia. Often, a decrease in blood glucose to $2.5 \mathrm{mmol} / \mathrm{L}$ or lower is not accompanied by clinical manifestations, or they are minor. On the other hand, symptoms such as dizziness, lethargy or aggression, weakness, sweating, trembling in the extremities, rapid pulse are not pathognomonic of HS, but can occur due to psycho-emotional agitation or as a result of auto-suggestion [1-4].

Thus, HS is a syndrome difficult to recognize with unclear definitions and a variety of causes and development mechanisms. Allocation of certain types of HS depends on the principle that was used for classification. Therefore, the author, using personal experience and a meta-analysis of thematic publications, offers two complementary classifications of HS - etiological, pathogenetic and clinical, which are presented below.

\subsection{Etiological and Pathogenetic Classification of Hypoglycemia}

1. Functional hypoglycemia: reactive hypoglycemia of the early phase, reactive hypoglycemia of the late phase, hunger glycogen-deficient hypoglycemia.

2. Exogenous hypoglycemia: associated with insulin therapy or oral sugar-lowering tablets, due to side effects of various "non-sugar reducing" drugs, toxigenic hypoglycemia, iatrogenic and autoiatrogenic hypoglycemia.

3. Hypoglycemia of an organic nature: adenoma or carcinoma of beta cells of islets of Langerhans, microadenomatosis of beta cells or their hyperplasia, paraneoplastic and pseudoparaneoplastic syndrome, acute and chronic pancreatitis.

4. Hypoglycemia in endocrine diseases: panhypopituitarism or selective hypopituitarism, hypoadrenocorticism (Addison's disease), hypothyroidism, thyrotoxicosis.

5. Hypoglycemia in somatic and systemic diseases (somatogenic): liver failure (non-tumor and nonenzymatic), chronic renal failure, chronic circulatory failure, encephalopathy of traumatic, vascular and other nature.

6. Autoimmune hypoglycemia (Hirat's disease).

7. Nonhypoglycemia and pseudohypoglycemia.

\subsection{Clinical Classification of Hypoglycemic Syndrome}

i. Fasting hypoglycemia.

1. Caused by drugs or toxigenic:

a) insulin, sulfonylurea preparations, salicylates, pentamidine, guanine;

b) alcohol.

2. Somatic diseases: liver, kidney disease, chronic heart failure, sepsis.

3. Hormone deficiency: cortisol, growth hormone, glucagon.

4. Tumor hypoclycemic syndrome:

a) pathology of beta cells; insulinoma, nesidioblastosis;

b) non-beta-cell tumors.

5. Autoimmune hypoglycemia (Hirat's disease):
a) antibodies to insulin;
b) antibodies to insulin receptors;
c) antibodies to beta-cells.

6. Pediatric hypoglycemia (derangement).

ii. Reactive hypoglycemia.

1. Congenital defect of enzymes involved in the metabolism of carbohydrates:

a) impaired fructose tolerance; 
b) galactosemia.

2. Digestive hypoglycemia.

3. Idiopathic (functional) post-food hypoglycemia.

\subsection{Epidemiology, Etiology and Pathogenesis of Hypoglycemia}

The frequency and incidence of HS cannot be determined, but it is obvious one of the most frequent emergency conditions. The so-called glucose tolerance test (GTT) reveals functional hypoglycemia in $25 \%$ of the studied subjects $[2,5]$. According to NADA, patients with diabetes suffer from hypoglycemia almost daily, and at least 1 time per year $\mathrm{HC}$ occurs. About $4 \%$ of deaths among patients with diabetes are caused by hypoglycemia. Only a few of the many forms of HS are relevant for the practitioner. These forms are considered below.

Enzymatic hypoglycemia is associated with a transient or persistent defect in the enzymes involved in energy metabolism. They are preferable to the infancy and childhood, and are rare.

Idiopathic functional hypoglycemia (FH). There are reactive $\mathrm{FH}$, associated with food intake, and hunger $\mathrm{FH}$, which develops after 6 or more hours after eating. Reactive FH usually has a mild, self-stopping course, and connected with unclear causative factor and pathogenetic mechanism. It is assumed that gastrointestinal motility in impaired intestinal tract, in particular after surgery, excessive activity of parietal digestion condition, the functional instability of the autonomic nervous system, the breakdown of regulatory mechanisms and insulin secretion, an increase in the number and / or affinity of insulin receptor, and possibly other factors [1-3]. In fact, FH is a kind of physiological norms, some form of not completely adequate reaction to food intake.

Fasting FH is more severe and, of course, is a pathologycal condition. It happens rarely. According to the pathogenesis, fasting FH is situational in nature, as it is caused by depletion of liver glycogen reserves during prolonged starvation, against the background of prolonged intense physical exertion, during pregnancy or lactation.

Exogenous hypoglycemia (EH) often occurs among patients with diabetes mellitus (DM) with insulin therapy, less often with oral hypoglycemic agents. The reasons are an overdose or inadequacy of antidiabetic agents, dietary errors, especially in terms of diet, alcohol abuse, excessive physical exertion. The type of diabetes and its duration, the presence of complications and concomitant diseases are important. HS among diabetics provokes infections, somatic diseases, mental and physical fatigue, certain medications intake. Such drugs include salicylates and other anti-inflammatory drugs, antibacterial sulfonamides and some antibiotics (chloramphenicol, tetracycline, metacycline), beta-blockers, psychotropic drugs (haloperidol, chlorpromazine), barbiturates, antihistamines, magnesium-containing drugs, PASK, quinine, anabolic steroids and a lot of other medicines $[3,6,7]$. It is important to remember that such drugs can cause glycopenia among patients without diabetes. Prescribing these drugs is not only for diabetics, but any patient should be controlled by the concentration of glucose in the blood, especially if there are signs of heart, liver or kidney failure.

Toxigenic hypoglycemia is a consequence of an overdose of certain drugs. Often this is poisoning with salicylates and barbiturates, usually for suicidal purposes. Toxigenic hypoglycemia is possible with an overdose of biguanides, which do not cause hypoglycemia in a therapeutic dose. Severe hypoglycemia complicates alcohol intoxication, or poisoning with nitro-varnishes and nitro-paints.

The term "iatrogenic or autoiatrogenic hypoglycemia" is used when the corresponding syndrome is caused by erroneous administration, or unjustified administration of insulin, or by taking sugar-lowering tablets. In this case hypoglycemia can complicate the infusion of a polarizing solution, or the administration of small doses of insulin for anabolic purposes. There are known cases of iatrogenic hypoglycemia as a result of applying insulin dressings for the treatment of sluggish wounds and trophic ulcers.

Autoiatrogenic hypoglycemia is associated with the deliberate, unreasonable use of hypoglycemic agents in order to cause a hypoglycemic state. This autoiatrogenism occurs in doctors and relatives of patients with diabetes mellitus. It is believed that this phenomenon is associated with mental disorders $[1,3,4]$.

Organic hypoglycemia is often called organic hyperinsulinism. It develops either as a result of increased insulin secretion by beta-cells of the islets of Langerhans, or due to ectopic formation of insulin by malignant tumors, including those not related to the endocrine glands.

Hyperinsulinism is usually associated with a beta-cell tumor of the pancreas - insulinoma. Less commonly, the syndrome is caused by hyperplasia (nezidioblastosis) or isletcell microadenomatosis $[8,9]$. It is important to remember that insulinoma often develops as part of multiple endocrine neoplasia (MEN- syndrome) [10].

Paraneoplastic hypoglycemia connected with malignant tumors, which sometimes secrete insulin or insulin-like substances with a hypoglycemic effect. Most often it is an insulin-like growth factor-II (IGF-II). Pseudoparaneoplastic hypoglycemia is considered a consequence of increased glucose uptake by a massive, rapidly growing tumor. Another hypothesis explains the pseudoparaneoplastic HS by the inhibition of gluconeogenesis in the liver and kidneys by the toxic products of the oncological process. It is likely that these mechanisms are combined [11, 12].

HS rarely complicates acute, even less often, chronic pancreatitis, which is explained by the massive release of insulin from damaged beta cells.

In endocrinopathies, HS is caused by the lack of one or several contra-insulin-hormones that balance the action of insulin through the processes of glycogenolysis, lipolysis, and gluconeogenesis. This hypoglycemia is always caused by hunger: develops either on an empty stomach, or 5-6 hours after eating. Such a variant of hypoglycemia may be accompanied by panhypopituitarism, selective hypopituitarism (isolated ACTH or STH deficiency), 
congenital adrenal hyperplasia syndrome, Addison's disease, hypothyroidism.

A special place among endocrinopathic HS has hypoglycemia with thyrotoxicosis. Typically, thyrotoxicosis is accompanied by a decrease in tolerance to carbohydrates or manifest diabetes, due to beta-adrenergic blockade of insulin secretion, enhanced glycolysis and lipolysis. But sometimes with thyrotoxicosis due to the accelerated metabolism of energy substrates and their depletion, hypoglycemia occurs.

Somatogenic hypoglycemia is a common but rarely recognized syndrome. Usually this is hepatopathyc hypoglycemia, the pathogenesis of which is associated with a decrease in gluconeogenesis in cirrhosis or fatty degeneration of the liver, chronic hepatitis, cholangitis or liver abscess.

A decrease in gluconeogenesis in the kidneys due to damage to the renal parenchyma, as well as against a background of poor nutrition due to intoxication and constant nausea and vomiting, are the causes of hypoglycemia in patients with chronic renal failure.

Hypoglycemia sometimes complicates heart failure syndrome, which is associated with a number of reasons. Firstly, poor health and lack of appetite lead to a deficiency of food substrates, depletion, a decrease in adipose tissue and glycogen stores. Secondly, gluconeogenesis in the liver and kidneys is disturbed due to their secondary dysfunction.

Cerebral or encephalopathic hypoglycemia, which occasionally complicates traumatic or vascular brain damage, has a different pathogenesis. In some cases, hypothalamic dysfunction with hypertonicity of $\mathrm{n}$. vagus is accompanied by vagus-insular paroxysms. In others, a contra-action occurs due to HG or ACTH deficiency, and cortical-adrenal insufficiency.

Autoimmune hypoglycemia or Hirat's disease is one of the rarest forms of HS, associated with the formation of antibodies to insulin, insulin receptors and pancreatic betacell antigens. Insulin antibodies sometimes appear in people who have never received insulin injections. These spontaneous antibodies block insulin, causing clinical and biochemical manifestations of diabetes. Occasionally, also for an unclear reason, the insulin-antibody complex disintegrates, with the release of insulin and development of severe HS.

Antibodies to insulin receptors can be blocking and stimulating. Depending on this, a diabetic or hypoglycemic syndrome is formed. Antibodies to islet antigens sometimes exhibit a temporary ability to stimulate insulin secretion. For this reason, in some patients, shortly before the manifestation of type 1 diabetes, attacks of hypoglycemia are noted [6].

Nonhypoglycemia means an involuntary error in the laboratory test on glucose in the blood. For example, false chemical hypoglycemia is often fixed in patients with leukemia, erythroblastosis, myeloma, and even with a banal leukocytosis, due to the inflammatory process of [1, 3, 4].

Pseudohypoglycemia is a psychopathological syndrome of the type of hysteria, with an imitation of subjective symptoms of hypoglycemia.

\subsection{The Clinical Picture and Diagnosis of Hypoglycemic Syndrome}

Concerning the semiotics of hypoglycemia, the main feature of HS is that its clinical symptoms are nonspecific, variable and poorly correlated with the concentration of glucose in the blood [2, 11-13].

The primary basis of the clinical symptoms of HS is dysfunction of the central nervous system (CNS) and the autonomous nervous system (ANS). It is known that the sensitivity of nerve structures to energy deficiency varies greatly, depending on their location, a peculiarity of subsystem and the function performed. It is generally accepted that the initial symptoms of hypoglycemia are caused by a disorder of the higher parts of the central nervous system, i.e. cortical functions. This includes a violation of the intellectual and behavioral spheres in the form of distraction, memory loss, irritability and anxiety, or, on the contrary, lethargy, drowsiness and apathy occur. These disorders are called early neuroglycopenic symptoms.

Simultaneously with these symptoms, activation of the ANS manifests itself. This is dizziness, headache, cold sweat, "rumbling" in the abdomen and a kind of discomfort in the epigastric region (sucks under the stomach), nausea and increased salivation, spastic abdominal pain, decreased vision and tinnitus, chills, sometimes feeling internal trembling, numbness and cooling of limbs. The listed phenomena are mainly associated with the activation of the sympatheticadrenal part of the ANS, therefore they are often called adrenergic symptoms of HS.

In fact, the neuroglycopenic and adrenergic manifestations of HS occur simultaneously, so the accepted separation of symptoms into the initial adrenergic and subsequent neuroglycopenic phases is not entirely correct.

A decrease in glycemia to a glycopenic level below 3 mmol / L is accompanied by dysfunction of more ancient cortical formations and diencephalic structures. The first are associated with psychopathological reactions such as stupor, disorientation, psychomotor agitation, inadequate speech and acts of an aggressive plan, hallucinations and short-term fainting. Diencephalic disorders are realized mainly by vegetative-neurotic symptoms - tremor of the hands, numbness of the lips, tongue and limbs, increased sweating and sawdust, hyperemia or pallor of the skin, heart palpitations, anisocoria, strabismus, nystagmus, increased tendon reflexes.

Subsequently, as glycopenia deepens, gross neurological symptoms appear - hyperkinesis (tonic and clonal convulsions, trismus), inhibition of tendon reflexes, paralysis of limb muscles, pathological foot reflexes, meningeal symptoms and symptoms of oral automatism. Then consciousness is lost, and hypoglycemic coma develops [13].

Symptoms of HS, given the variety of causal factors and other conditions, are variable and unpredictable. The clinical mask of hypoglycemia may look like delirium, reactive psychosis (panic-attack), an attack of epilepsy or hysteria, vegetative-vascular paroxysm, myasthenic crisis, acute 
neuromyopathy, stroke, pseudo-organic neurological syndrome that mimics a tumor of the brain or spinal cord [1, $2,5,13]$. These phantoms are especially characteristic of elderly and senile people, in whom HS represents a great danger in terms of serious consequences. Therefore, the diagnosis of HS based on only of complaints and physical data is impossible.

It is no coincidence that with coma of unclear origin or with reactive conditions, it is recommended a priori to administer $40 \mathrm{ml}$ of a $40 \%$ glucose solution intravenously, which serves both as emergency therapy and as an ex juvantibus diagnosis.

\subsection{Emergency Treatment of HC and Pathogenetic Diagnosis of $\mathrm{HS}$}

Urgent therapy of $\mathrm{HC}$ is presented in Table 1.

Table 1. Urgent therapy of hypoglycemic coma.

\begin{tabular}{|c|c|c|}
\hline Primary Therapy & Syndrome Therapy & Diagnosis \\
\hline \multicolumn{3}{|l|}{ First $5-10$ minutes } \\
\hline $\begin{array}{l}\text { Intravenously, } 40-80 \mathrm{ml} \text { jet } 40 \% \text { glucose solution } \\
\text { If venipuncture is impossible, intramuscularly } \\
1 \mathrm{mg} \text { glucagon, or subcutaneously } 0.5-1.0 \mathrm{ml} 1 \% \text { sol. } \\
\text { adrenaline hydrochloride }\end{array}$ & $\begin{array}{l}\text { In case of loss of consciousness, especially } \\
\text { with convulsions, take measures against } \\
\text { aspiration and trauma } \\
\text { Urgent hospitalization in the ward of intensive } \\
\text { treatment }\end{array}$ & $\begin{array}{l}\text { Express-test on blood glucose } \\
\text { Control of hemodynamic }\end{array}$ \\
\hline \multicolumn{3}{|l|}{ Next $10-15$ minutes } \\
\hline $\begin{array}{l}\text { 1. If consciousness is not restored, } 40-80 \mathrm{ml} \text { of } 40 \% \\
\text { glucose solution is re-injected into a vein } \\
\text { 2. If there is still no consciousness, intravenously drip } \\
500 \mathrm{ml} \text { of } 10 \% \text { glucose solution }\end{array}$ & $\begin{array}{l}\text { With severe headache, analgesics, No-Spa, } \\
\text { Novigan } \\
\text { With nausea and vomiting: make an injection } \\
\text { of Cerucal, Zofran, Latran }\end{array}$ & $\begin{array}{l}\text { Electrocardiogram } \\
\text { Consultation of a oculist and neurologist for } \\
\text { stroke and } \\
\text { cerebral oedema }\end{array}$ \\
\hline \multicolumn{3}{|l|}{ After 30 minutes } \\
\hline $\begin{array}{l}\text { Glycemia }>5 \mathrm{mmol} / \mathrm{L} \text {, but consciousness has not } \\
\text { recovered. There are signs of cerebral oedema: } \\
\text { intravenously, drip } 500 \mathrm{ml} \text { of a } 20 \% \text { mannitol } \\
\text { solution; } \\
\text { into the stomach through a tube } \\
300 \mathrm{ml} \text { of glycerol; } \\
\text { intravenously } 4-8 \mathrm{mg} \text { of dexamethasone; } \\
\text { intravenously } 40 \mathrm{mg} \text { Lasix. }\end{array}$ & $\begin{array}{l}\text { Oxygen } \\
\text { According to the testimony of hypotensive, } \\
\text { analeptic, cardiotonic, etc. }\end{array}$ & $\begin{array}{l}\text { If cerebral oedema, stroke, and cardiogenic } \\
\text { shock are excluded, and glycemia }>5 \mathrm{mmol} / 1 \text {, } \\
\text { reactive psychosis or hysteria should be } \\
\text { suspected. Urgent inspection of } \\
\text { a psychiatrist. }\end{array}$ \\
\hline
\end{tabular}

Note: glucagon is not used for HS associated with sulfonylurea preparations, since glucagon additionally stimulates insulin secretion. A glucose solution is administered intravenously with octreotide, which suppresses the secretion of insulin.

After stopping the patient's syndrome, they are hospitalized, because HS are fraught with complications, moreover, most types of hypoglycemia tend to recur. The nature of the HS is clarified using a series of tests and samples (Figures 1-3).

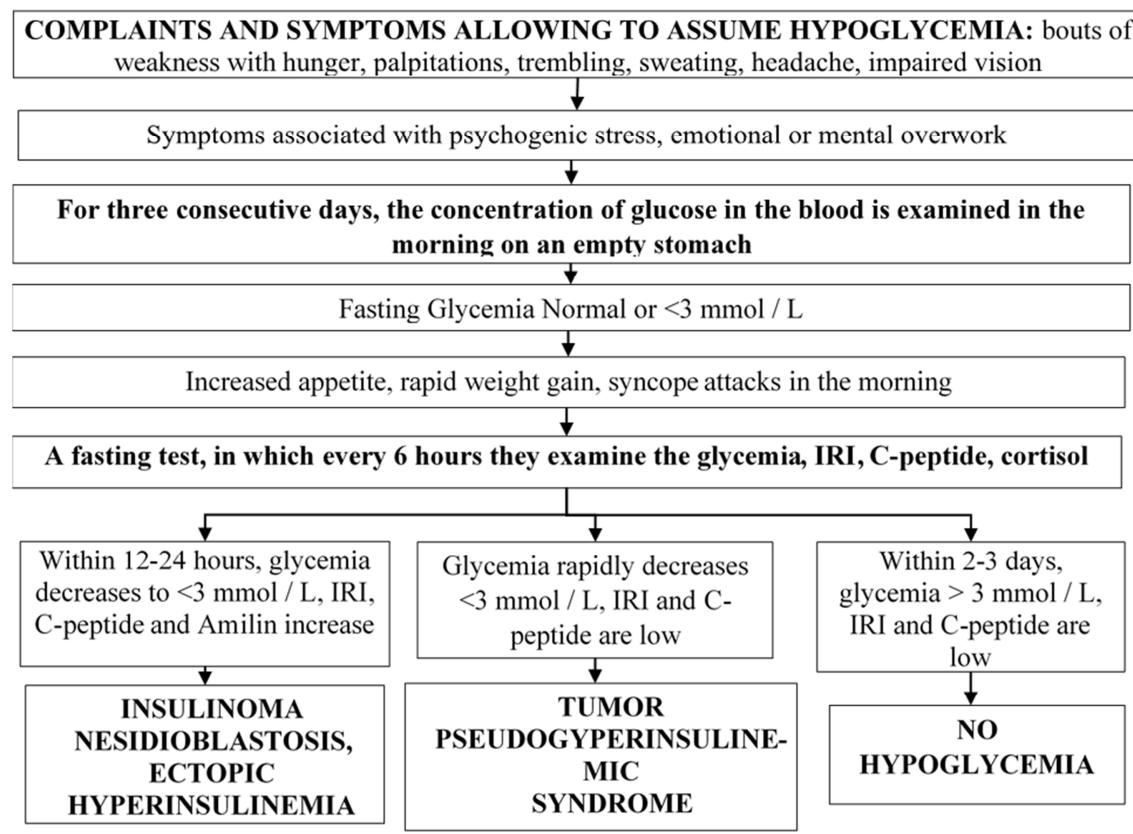

Figure 1. "Diagnosis of hypoglycemic syndrome". 


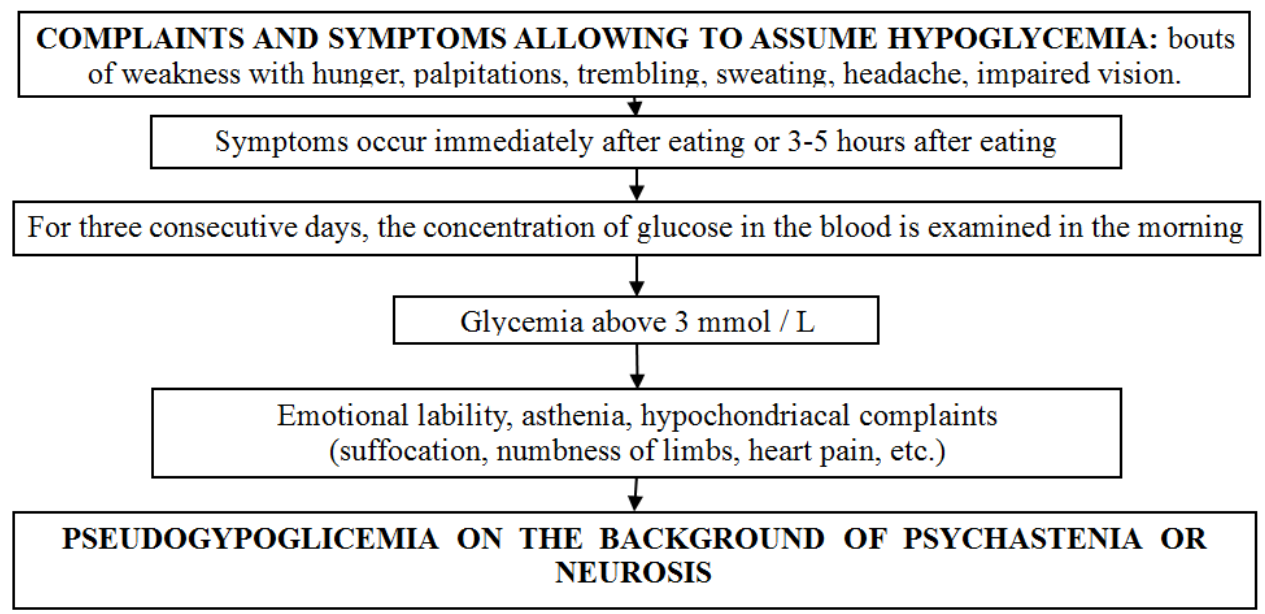

Figure 2. Diagnosis of hypoglycemic syndrome.

\section{COMPLAINTS AND SYMPTOMS ALLOWING TO ASSUME HYPOGLYCEMIA}

attacks of weakness with hunger. palpitations, trembling. sweating. headache. impaired vision.

\begin{tabular}{|c|c|c|c|}
\hline \multicolumn{4}{|c|}{$\begin{array}{l}\text { For three consecutive days, the concentration of glucose in the blood is examined in the } \\
\text { morning on an empty stomach }\end{array}$} \\
\hline & \multicolumn{3}{|c|}{ In individual samples, glycemia $<3 \mathrm{mmol} / \mathrm{L}$} \\
\hline \multicolumn{4}{|c|}{ Obesity, a history of gastrointestinal surgery, intolerance to certain foods } \\
\hline \multicolumn{2}{|c|}{$\downarrow$} & \multicolumn{2}{|c|}{$\downarrow$} \\
\hline \multicolumn{2}{|c|}{$\begin{array}{l}\text { OGTT ( } 75 \mathrm{~g} \text { glucose) every } 30 \mathrm{~min} \text {. within } 3 \\
\text { hours in the blood determine glucose, IRI and }\end{array}$} & \multicolumn{2}{|c|}{ Fermentological tests } \\
\hline$\checkmark$ & & \multirow{2}{*}{\multicolumn{2}{|c|}{$\begin{array}{l}\text { Deficiency of glucosotropic } \\
\text { Enzymes. Drugs or other } \\
\text { substances in a blood }\end{array}$}} \\
\hline $\begin{array}{l}\text { Glycemia }<3 \mathrm{mmol} / \\
\mathrm{L}, \text { IRI and cortisol } \\
\text { sharply increased }\end{array}$ & $\begin{array}{l}\text { Glycemia }>3 \mathrm{mmol} / \\
\mathrm{L}, \text { IRI and cortisol } \\
\text { grow moderately }\end{array}$ & & \\
\hline$\downarrow$ & $\downarrow$ & $\sqrt{ }$ & $\neg$ \\
\hline $\begin{array}{c}\text { REACTIVE } \\
\text { HYPOGLYCAEMIA }\end{array}$ & $\begin{array}{c}\text { NO REACTIVE } \\
\text { HYPOGLYCEMIA } \\
\end{array}$ & $\begin{array}{c}\text { ENZYME } \\
\text { DEFICIENCY } \\
\text { HYPOGLYCEMIA }\end{array}$ & $\begin{array}{l}\text { AUTOYATROGE- } \\
\text { NIC } \\
\text { HYPOGLYCEMIA }\end{array}$ \\
\hline
\end{tabular}

Figure 3. Diagnosis of hypoglycemic syndrome.

\subsection{Methods of Laboratory Diagnostics}

Determination of glucose in capillary blood. It is better to use enzymatic methods for testing glycemia - glucokinase or glucose oxidase. In practice, the orthotoluidine method is used, which provides sufficiently accurate and reproducible data. But we must remember that the orthotoluidine method gives overestimated numbers in patients with chronic renal failure, or after infusion of colloidal plasma substitutes [12, 14].

Hormone tests and combined indexes. In $15-20 \%$ of patients with insulinoma and other forms of organic hypoglycemia, fasting glycemia is normal. Therefore, together with glycemia, immunoreactive insulin (IRI) is determined in the blood and the index "IRI / glycemia" is calculated (see below) [15]. If iatrogenic hyperinsulinemia is suspected, the level of C-peptide in the blood is determined, IGF-II is determined for the diagnosis of paraneoplastic condition. However, the diagnostic search for HS requires many other hormone and metabolic tests.

A 3-hour oral glucose tolerance test (OGTT) is performed not earlier than $10 \mathrm{am}$ and no later than $4 \mathrm{pm}$, at least 7 hours after the last meal. Before the test and during the test, the patient should not smoke, worry, move, drink water and eat.

An adult drinks $250-300 \mathrm{ml}$ of weak tea with 75 grams of glucose in 5 minutes. The child is given glucose per $1 \mathrm{~g}$ per $\mathrm{kg}$ of body weight. Before taking glucose, the blood glucose level is determined, then glycemia is monitored every 30 or 60 minutes. The test is positive if, in one of the definitions 
(excluding the first, before glucose intake), glycemia decreases by $50 \%$ or more from the initial one, and clinical symptoms of HS appear. The test is most informative in the diagnosis of functional (reactive) hypoglycemia.

Glucagon test. Intravenously, within 2-3 minutes, glucagon is administered at the rate of 30 microgram per $\mathrm{kg}$ of weight (but not more than 1 milligram). Prior to administration and every 5 minutes after administration of glucagon, the concentration of glucose, IRI, C-peptide in the blood is determined, and in cases of suspected Girke's disease or alcoholic hypoglycemia lactic acid is tested. Normally, glycemia increases at least twice, and IRI 2-3 times compared with the initial level. With insulinoma, an increase in the circulating level of IRI is more pronounced and is usually accompanied by rebound hypoglycemia.

Calcium Infusion Test. 10\% solution of calcium lactate, gluconate or carbonate at a dose of $10 \mathrm{mg} \mathrm{Ca}^{2+}$ per $\mathrm{kg}$ of body weight is infused intravenously, dropwise, over 2 hours. Before and after infusion the concentration of glucose and IRI is determined in the blood. Normally, a decrease in glycemia and an increase in IRI is not more than $10-15 \%$ of the initial one. With insulinoma, clinical and chemical hypoglycemia develops, and IRI increases several times.

Test with fasting. The hunger test is used for the differential diagnosis of reactive and organic hypoglycemia. At the beginning of the sample and then every hour, the concentration of glucose, IRI and C-peptide in the blood is determined. During the test a patient is continuously monitored by a doctor or nurse, who must be able to stop hypoglycemic paroxysm. Approximately half of patients with insulinoma develop hypoglycemia already in the first 3-4 hours, while the rest have a hypoglycemic attack on average 24 hours later, although cases have been described when a patient with insulinoma does not have hypoglycemia even after 72 hours from the onset of fasting [2, 3, 11]. The information content of the sample increases if, simultaneously with glycemia, IRI and C-peptide are examined.

Data is calculated as the indices "IRI / glycemia" and "Cpeptide / glycemia" [15]. Normally, the IRI index $\mu \mathrm{U} / \mathrm{ml} /$ Glycemia mmol / L does not exceed 5. Before the breakdown, the indices are increased for many types of HS and even for simple obesity. But during the test in patients with insulinoma, the indices are steadily increasing, and with other forms of HS decrease.

A more sensitive analogue of the IRI / Glycemia index is the Turner's Index (TI), calculated by the following formula:

$\mathrm{TI}=[$ Plasma insulin $(\mu \mathrm{U} / \mathrm{ml}) \times 100) /$ Plasma glucose $(\mathrm{mg} \%)]-30$.

The value of TI in healthy people is below 30 , in people with obesity about 100 , in patients with insulinoma - above 150 .

\section{Discussion}

In the last 2-3 decades, ideas about the causes and mechanisms of the development of hypoglycemic syndrome have been significantly enriched and updated. The list of HS forms has been expanded, and the classifications of this syndrome have acquired a more detailed, practically oriented character $[2,6,11,16]$. The pathophysiology was clarified and the recognition of HS in somatic and endocrine diseases, enzymopathy, and iatrogenism was facilitated, which is largely explained by the increased capabilities of laboratory, functional, and introscope diagnostics $[8,11,14,15]$. At the same time, with regard to new or improved laboratory tests, as well as modern imaging techniques for insulinoma and other tumors, the researchers' opinion is unambiguously positive [3, $8,11,14]$, while the reviews are negative for functional diagnostics. The point of view is expressed that informativeness of functional, especially pharmacodynamic tests is small, and the risks associated with such tests are high $[11,14,15]$. In this regard, there is a tendency to abandon the use of pharmacodynamic tests in the endocrinology clinic [12].

At the same time, it is obvious that a negative assessment of pharmacodynamic tests cannot be applied to a hunger test and an oral glucose tolerance test, since these tests are basic for the diagnosis of insulinoma and functional hypoglycemia [2].

Given the certain limitations that has the accuracy of laboratory identification of HS, and taking into account the huge variety of clinical phantoms of hypoglycemia, it should be recognized that emergency diagnosis of this syndrome is difficult. At the same time, not only the depth of hypoglycemia, but also its duration are risk factors for possible complications, therefore, the actual approach to HS, from the point of view of a practical doctor, is expressed by the phrase "Periculum in mora". In this regard, the recommendation seems to be quite rational, according to which a patient with twilight consciousness or in a coma should be given a priori intravenous, stream, 20-40 $\mathrm{ml}$ of a $40 \%$ glucose solution $[13,14,16]$.

\section{Conclusion}

In conclusion, it should be emphasized once again that HS and $\mathrm{HC}$ are associated with the danger of serious complications - hemorrhagic and circulatory disorders of cerebral blood flow, cerebral edema, pituitary apoplexy, and even stem disorders with the death of the patient. The most at risk are middle-aged and elderly. Many of them suffer from diabetes, which is often complicated by hypoglycemia. In this regard, hypoglycemia should be considered as a lifethreatening condition and requires emergency diagnosis and emergency care. Timely and accurate causal and pathogenetic diagnosis of hypoglycemia prevents the development of severe and dangerous HS and is an effective prevention of HC. Obviously, it is to this syndrome that the expression "Periculum in mora" is most applicable.

\section{References}

[1] Marks V., Rose F. Hypoglycemia. 2-nd ed. Blackwell Scientif. publ. N-Y: - 1981. 
[2] Lukyanchikov V. S., Endocrine Comas and Crises. M.: Academic Project. 2013. (in Rus.).

[3] Shuvin Z., Felig F. Hypoglycemia. In: Endocrinology and Metabolism (transl. from English). Ed. F. Felig et al. M.: Medicine. - 1985. - T. 2. - P. 218-258. (in Rus.).

[4] Yager J., Young R. Non-hypoglycemia is an epidemic condition. // New Engl. J. Med. - 1974. - № 14. - P. 507-508.

[5] Inzucchi S., Kinder B. Endocrine Emergencies: Hypoglycemic and Hyperglycemic Crises. In: Textbook of Endocrine Surgery. (Eds. O. Ciark, Q-Y. Duh.). / WE Sounders Company. Washington etc.: - 1997. - P. 175-181.

[6] Boyle P. J. Hypoglycemia. In: Medical Management of Diabetes Mellitus (transl. from English). By J. Leaty etc. (Eds). Marcel Dekker Inc. NY, Basel. 2000. Ch. 31.

[7] Ostroumova O. D., Akimova ES, Kochetkov A. I., Pereverzev A. P. Drug-induced hypoglycemia: a focus on drugs not belonging to the group of sugar-lowering drugs. // CONSILIUM Medicum. 2019. - T. 21. - No. 4. - P. 59-65. (in Rus.).

[8] Foster D., Rubinstein A. Hypoglycemia, insulinoma and other hormone-active tumors of the pancreas. In the book: Internal Diseases (transl. from English). ed. E. Braunwald and others M.: - Medicine. - 1997. - T. 9. - P. 232-247. (in Rus.).

[9] Kuzin N. M., Egorova L. V., Lakreeva MG. Gurevich L. E. Organic hyperinsulinism. // Clinical medicine. - 1998.- No. 4. - P. 7-11. (in Rus.).
[10] Rostomyan L. G., Rozhinskaya L. Ya., Tyulpakov A. N. Clinical and genetic characteristics of the syndrome of multiple endocrine neoplasia type 1 and the principles of its treatment. // Farmtek. - 2010. - No. 3. - P. 50-56. (in Rus.).

[11] Shustov S. B., Halimov U. Sh., Saluhov V. V., Trufanov G. E. Functional and Topical Diagnostic in Endocrinology. M.: GEOTAR Media. 2017. (in Rus.).

[12] Zilber A. P. Sketches of Critical Medicine. M.: Med press Inform. 2006. (in Rus.).

[13] Stefansson K., Arnason B. Neurological manifestations of systemic neoplasia. / In the book: Internal diseases. (translated from English). By E. Braunwald et al. (Eds.) M.: - Medicine. 1996. - T. 8. - P. 123-131. (in Rus.).

[14] Kamyshnikov V. S. Clinical laboratory tests from A to Z and their diagnostic profiles. / M.: - Medpress-Inform. - 2018. (in Rus.).

[15] Koryachkin V. A. Formulas and Calculations in intensive Therapy. SPb.: GMU. 2000. (in Rus.).

[16] Intensive Therapy. Contemporary Aspects. (transl. from English) By D. Sew, D. Wing (Eds.). M.: Medpress - Inform. 2008. (in Rus.). 